\title{
Challenging the weak cosmic censorship conjecture with charged quantum particles
}

\author{
Maurício Richartz, ${ }^{1, *}$ and Alberto $\mathrm{Saa}^{2, \text {, }}$ \\ ${ }^{1}$ Instituto de Física Teórica, Universidade Estadual Paulista, \\ Rua Dr. Bento Teobaldo Ferraz, 271 - Bl. II, 01140-070, São Paulo, SP, Brazil. \\ ${ }^{2}$ Departamento de Matemática Aplicada, UNICAMP, 13083-859 Campinas, SP, Brazil.
}

\begin{abstract}
Motivated by the recent attempts to violate the weak cosmic censorship conjecture for nearextreme black-holes, we consider the possibility of overcharging a near-extreme Reissner-Nordström black hole by the quantum tunneling of charged particles. We consider the scattering of spin-0 and spin- $\frac{1}{2}$ particles by the black hole in a unified framework and obtain analytically, for the first time, the pertinent reflection and transmission coefficients without any small charge approximation. Based on these results, we propose some gedanken experiments that could lead to the violation of the weak cosmic censorship conjecture due to the (classically forbidden) absorption of small energy charged particles by the black hole. As for the case of scattering in Kerr spacetimes, our results demonstrate explicitly that scalar fields are subject to (electrical) superradiance phenomenon, while spin- $\frac{1}{2}$ fields are not. Superradiance impose some limitations on the gedanken experiments involving spin-0 fields, favoring, in this way, the mechanisms for creation of a naked singularity by the quantum tunneling of spin- $\frac{1}{2}$ charged fermions. We also discuss the implications that vacuum polarization effects and quantum statistics might have on these gedanken experiments. In particular, we show that they are not enough to prevent the absorption of incident small energy particles and, consequently, the formation of a naked singularity.
\end{abstract}

PACS numbers: 04.70.Dy, 04.20.Dw, 04.62.+v

\section{INTRODUCTION}

The weak cosmic censorship conjecture (WCCC), proposed by Roger Penrose in 1969 , asserts that any singularity originated from gravitational collapse must be hidden inside the event horizon of a black hole 1]. Even though the WCCC is a necessary condition to guarantee the predictability of classical General Relativity, its validity remains an open question. Since it has been proposed, numerous classical results have given strong support to the conjecture 2, 3]. For example, if the WCCC is false, then it would be natural to expect that the formation of a black hole in gravitational collapse would be a non-generic outcome. However, the fact that stationary black holes are stable under linear perturbations provides good evidence in favor of the conjecture. Another typical test of the WCCC consists on gedanken experiments trying to destroy the event horizon of a black hole and consequently exposing its inner singularity to an outside observer. These thought experiments are based on the uniqueness theorems that assert that all stationary black hole solutions of the Einstein-Maxwell equations are characterized by three conserved parameters: the gravitational mass $M$, the electric charge $Q$ and the angular momentum $J$, which satisfy (we assume geometrized units, so that $c=G=\hbar=1$ )

$$
M^{2} \geq Q^{2}+\left(\frac{J}{M}\right)^{2}
$$

Solutions whose parameters do not satisfy (1) are generically dubbed naked singularities. When the equality holds, the black hole is called extreme. We call a black hole near-extreme if $0<M^{2}-Q^{2}-(J / M)^{2} \ll M^{2}$.

The basic idea behind the most common gedanken experiments is to make a black hole absorb a test particle with enough charge and/or angular momentum so that condition (1) ceases to be valid. Wald [2] has shown that, if the black hole is initially extreme, its event horizon is always preserved because the particle total energy required to surpass the potential barrier (created by the interaction particle-hole) more than compensates the increase in charge and/or angular momentum. We can also conclude from Wald's pioneering work that quasistationary processes cannot be used to destroy the event horizon of a black hole since, in such processes, the black hole would be arbitrarily close to extremality before becoming a naked singularity. Some other classical analysis, however, indicate that a violation of the WCCC is possible if we start with a near-extreme black hole but use non-stationary processes instead [4 7] (see also Ref. [8]). Since the destruction of the event horizon, in these cases, occurs due to feeble effects beyond the linear first approximation, a way to avoid the formation of naked singularities might be to consider higher order effects, e.g. self-energy corrections of the particle's total energy [46, $9,[10]$.

Some interesting recent attempts to destroy the event horizon are based on quantum tunneling of particles into near-extreme black holes. The original idea, by Matsas and Silva [11], is based on the fact that, due to the waveparticle duality, the tunneling probability can be nonzero even if the particle total energy is smaller than the height of the potential barrier, indicating a possible vio-

* richartz@ift.unesp.br

† asaa@ime.unicamp.br 
lation of the WCCC. However, since we lack a complete description of the quantum gravity regime, a final conclusion cannot yet be obtained, see Ref. [9 14] for further details and considerations.

So far, only neutral particles have been treated from this quantum perspective. In this paper, we will consider the tunneling process of charged spin- 0 and spin- $\frac{1}{2}$ particles into a charged black hole. We set up a unified framework for describing scattering processes of these particles by Reissner-Nordström $(J=0)$ black holes and evaluate analytically the pertinent transmission and reflection coefficients for all possible values of charge. To the best of our knowledge, this has only been done before in the limit of small charges, see Ref. [15, 16]. The calculations are performed in the limit of interest for violating the WCCC: the limit of small energies for incident massless particles. Based on these scattering processes, we propose some gedanken experiments that would be able, in principle, to violate the WCCC due to quantum tunneling of charged particles with very small energies (not sufficient to preserve (1) after the absorption by the black hole). Interestingly enough, we have, as for the case of Kerr spacetimes, that spin- $\frac{1}{2}$ fermions are not subject to the (electrical) superradiance phenomenon, suggesting that such fermions are the best candidates for the creation of naked singularities in gedanken experiments involving quantum tunneling processes. In fact, we will show that superradiance effectively makes more difficult the formation of a naked singularity by the absorption of a scalar charged particle by the black hole. We also consider some vacuum polarization and quantum statistics effects that might influence these processes, and we show that they are not enough to prevent the formation of a naked singularity.

\section{CHARGED FIELDS AROUND A REISSNER-NORDSTRÖM BLACK HOLE}

A non-rotating charged black hole of mass $M$ and charge $Q$ corresponds to the Reissner-Nordström (RN) spacetime. By using spherical coordinates, the RN metric can be cast as

$$
d s^{2}=-\frac{\Delta}{r^{2}} d t^{2}+\frac{r^{2}}{\Delta} d r^{2}+r^{2}\left(d \theta^{2}+\sin ^{2} \theta d \varphi^{2}\right),
$$

where

$$
\Delta=r^{2}-2 M r+Q^{2}=\left(r-r_{+}\right)\left(r-r_{-}\right) .
$$

The roots of $\Delta$, denoted by $r_{+}$and $r_{-}$, are, respectively, the event horizon and the Cauchy horizon of the black hole. Notice that the extreme case corresponds to $r_{+}=r_{-}$, whereas for a naked singularity, i.e. solutions for which condition (1) does not hold, $\Delta$ has no real root. The only non-vanishing component of the electromagnetic potential $A_{\mu}$ for the RN metric is $A_{0}=-Q / r$. Charged spin-0 and spin- $\frac{1}{2}$ fields on the RN metric (2) can be described effectively in the unified framework that we present here.

\section{A. Massless charged scalar field}

A massless charged scalar field propagating in the $\mathrm{RN}$ background is described by the usual Klein-Gordon equation [17],

$$
\left(\nabla_{\mu}-i q A_{\mu}\right)\left(\nabla^{\mu}-i q A^{\mu}\right) \phi=0,
$$

where $q$ stands for the electric charge of the scalar field. Note that the usual covariant derivative operator has been replaced by $\nabla_{\mu}-i q A_{\mu}$ to include the minimal coupling between the RN electromagnetic potential and the charge of the field. This equation can be easily separated in the $\mathrm{RN}$ metric if we consider the ansatz

$$
\phi=e^{-i \omega t} e^{i m \varphi} S_{0}(\theta) R_{0}(r) .
$$

The resulting radial and angular equations will be given by the forthcoming master equations (17) and (18) with $s=0$.

\section{B. Massless charged spin- $\frac{1}{2}$ field}

In the Newman-Penrose formalism, the wave function describing a massless charged spin- $\frac{1}{2}$ field is represented by a pair of spinors, $P^{A}$ and $\bar{Q}^{A}$, which obey the Dirac equations

$$
\begin{aligned}
& \sigma_{A B^{\prime}}^{\mu}\left(\nabla_{\mu}-i q A_{\mu}\right) P^{A}=0, \\
& \sigma_{A B^{\prime}}^{\mu}\left(\nabla_{\mu}+i q A_{\mu}\right) Q^{A}=0,
\end{aligned}
$$

where $\sigma^{\mu} A B^{\prime}$ stands for the generalizations of the Pauli spin matrices called Infeld-van der Waerden symbols [18]. Minimal coupling is also assumed in this case. The following transformations,

$$
\begin{aligned}
P^{0} & =\frac{R_{-\frac{1}{2}}(r) S_{-\frac{1}{2}}(\theta)}{r} e^{-i \omega t} e^{i m \varphi}, \\
P^{1} & =R_{+\frac{1}{2}}(r) S_{+\frac{1}{2}}(\theta) e^{-i \omega t} e^{i m \varphi}, \\
\bar{Q}^{1^{\prime}} & =R_{+\frac{1}{2}}(r) S_{-\frac{1}{2}}(\theta) e^{-i \omega t} e^{i m \varphi}, \\
\bar{Q}^{0^{\prime}} & =-\frac{R_{-\frac{1}{2}}(r) S_{+\frac{1}{2}}(\theta)}{r} e^{-i \omega t} e^{i m \varphi},
\end{aligned}
$$

can be used to separate the Dirac equations (6) and (7). The resulting expressions are [19, 20]

$$
\begin{aligned}
\Delta^{\frac{1}{2}}\left(\partial_{r}-i \frac{K}{\Delta}\right) R_{-\frac{1}{2}} & =\lambda \sqrt{\frac{\Delta}{2}} R_{+\frac{1}{2}}, \\
\Delta^{\frac{1}{2}}\left(\partial_{r}+i \frac{K}{\Delta}\right)\left(\sqrt{\frac{\Delta}{2}} R_{+\frac{1}{2}}\right) & =\lambda R_{-\frac{1}{2}},
\end{aligned}
$$

for the radial part and

$$
\begin{aligned}
& \left(\partial_{\theta}+m \csc \theta+\frac{1}{2} \cot \theta\right) S_{+\frac{1}{2}}=-\lambda S_{-\frac{1}{2}}, \\
& \left(\partial_{\theta}-m \csc \theta+\frac{1}{2} \cot \theta\right) S_{-\frac{1}{2}}=\lambda S_{+\frac{1}{2}},
\end{aligned}
$$


for the angular one, where $\lambda$ is a separation constant and

$$
K=\omega r^{2}-q Q r
$$

It is possible to eliminate $R_{+\frac{1}{2}}$ (or, equivalently, $R_{-\frac{1}{2}}$ ) from the equations (12) and (13), in order to obtain an equation for $R_{-\frac{1}{2}}$ (or $R_{+\frac{1}{2}}$ ) only. A similar procedure can be made for the angular functions. The resulting equations are given by the master equations (17) and
(18) below, with $s= \pm 1 / 2$.

\section{THE SCATTERING}

The previous radial and angular equations for spin-0 and spin- $\frac{1}{2}$ particles can be written in a unified manner as single master equations (analogous to the Teukolsky equations for uncharged massless fields in the Kerr metric 21]). For the radial part, one has

$$
\Delta^{-s} \frac{d}{d r}\left(\Delta^{s+1} \frac{d R_{s}}{d r}\right)+\left(\frac{K^{2}-2 i s(r-M) K}{\Delta}+4 i s \omega r-2 i s q Q-\lambda_{s}\right) R_{s}=0
$$

where $\lambda_{s}$ is a separation constant, $\omega$ is the energy of the particle and $K$ is given by (16), while for the angular part we have

$$
\frac{1}{\sin \theta} \frac{d}{d \theta}\left(\sin \theta \frac{d S_{s}}{d \theta}\right)+\left(s+\lambda_{s}-\frac{m^{2}}{\sin ^{2} \theta}-\frac{2 m s \cos \theta}{\sin ^{2} \theta}-s^{2} \cot ^{2} \theta\right) S_{s}=0 .
$$

Analogously to the Teukolsky equations, the fact that the solutions of the angular equation (18) must be regular at $\theta=0$ and $\theta=\pi$, transforms (18) into a Sturm-Liouville problem for the separation constant $\lambda_{s}$ (which differs from the separation constant $\lambda$ ). The eigenfunctions which solve the problem are the spin-weighted spherical harmonics ${ }_{s} Y_{j}^{m}$, with corresponding eigenvalues given by $\lambda_{s}=(j-s)(j+s+1)$ [22]. The quantities $-|\ell-| s|| \leq j \leq \ell+|s|, \ell$, and $-\ell \leq m \leq \ell$ are, respectively, the total, orbital and azimuthal angular momenta of the particle. Defining a new (tortoise) radial coordinate $r_{*}$ by $d r_{*} / d r=r^{2} / \Delta$ and a new function $Y_{s}=\Delta^{s / 2} r R_{s}$, the radial equation (17) can be written as

$$
\frac{d^{2} Y_{s}}{d r_{*}}+V_{s}\left(r_{*}\right) Y_{s}=0
$$

where

$$
V_{s}\left(r_{*}\right)=\frac{\Delta}{r^{4}}\left[\frac{(K-i s(r-M))^{2}}{\Delta}+4 i s \omega r-2 i s q Q-j(j+1)+s^{2}-2 \frac{M}{r}+2 \frac{Q^{2}}{r^{2}}\right] .
$$

Let us now consider the scattering of an incident wave originated far away from the black hole. This process can be described by a solution $Y_{s}$ of the wave equation with the following asymptotic behavior far from the black hole (see the Appendix A for the calculation details)

$$
Y_{s} \rightarrow Z_{s}^{\text {in }} r_{*}^{s+i q Q} e^{-i \omega r_{*}}+Z_{s}^{\text {out }} r_{*}^{-s-i q Q} e^{+i \omega r_{*}},
$$

and the following asymptotic behaviour near the event horizon of the black hole,

$$
Y_{s} \rightarrow Z_{s}^{\operatorname{tr}} e^{-\frac{s}{2}\left(\frac{r_{+}-r_{-}}{r_{+}^{2}}\right) r_{*}-i\left(\omega-\frac{q Q}{r_{+}}\right) r_{*}} .
$$

The $Z_{s}^{\text {in }}, Z_{s}^{\text {out }}$ and $Z_{s}^{\text {tr }}$ coefficients correspond, respectively, to the incident, reflected and transmitted wave amplitudes. Note that, in the fermionic case, $Z_{+\frac{1}{2}}$ and $Z_{-\frac{1}{2}}$ are not independent. For such a case, plugging the asymptotic forms (21) and (22) into Eqs. (12) and (13), we obtain

$$
\begin{aligned}
2 i \omega Z_{+\frac{1}{2}}^{\text {out }} & =\star \sqrt{2} Z_{-\frac{1}{2}}^{\text {out }}, \\
2 \sqrt{2} i \omega Z_{-\frac{1}{2}}^{\text {in }} & =-\lambda Z_{+\frac{1}{2}}^{\text {in }}, \\
\frac{\lambda}{\sqrt{2}} Z_{+\frac{1}{2}}^{\mathrm{tr}} & =\left(\frac{1}{2}-2 i \delta\right)\left(r_{+}-r_{-}\right)^{1 / 2} Z_{-\frac{1}{2}}^{\mathrm{tr}},
\end{aligned}
$$

where $\delta$ is given by

$$
\delta=\frac{r_{+}^{2}}{r_{+}-r_{-}}\left(\omega-\frac{q Q}{r_{+}}\right) .
$$

In order to derive relations between the (in/out/tr) coefficients, we use the fact that the Wronskian $W$ of two linearly independent solutions of the radial equation is independent of $r$,

$$
W\left[Y_{s}, Y_{-s}^{*}\right]_{r=r_{+}}=W\left[Y_{s}, Y_{-s}^{*}\right]_{r=\infty} .
$$


Using the solution whose asymptotic behaviour is given by Eqs. (21) and (22), we obtain the relation

$$
r_{s}+t_{s}=1
$$

where

$$
r_{0}=\left|\frac{Z_{0}^{\text {out }}}{Z_{0}^{\text {in }}}\right|^{2}, \quad t_{0}=\frac{1}{\omega}\left(\omega-\frac{q Q}{r_{+}}\right)\left|\frac{Z_{0}^{\text {tr }}}{Z_{0}^{\text {in }}}\right|^{2},
$$

for the spin-0 case and

$$
r_{\frac{1}{2}}=4 \frac{\omega^{2}}{\lambda^{2}}\left|\frac{Z_{+\frac{1}{2}}^{\text {out }}}{Z_{+\frac{1}{2}}^{\text {in }}}\right|^{2}, \quad t_{\frac{1}{2}}=\frac{\sqrt{r_{+}-r_{-}}}{r_{+}^{2}}\left|\frac{Z_{+\frac{1}{2}}^{\operatorname{tr}}}{Z_{+\frac{1}{2}}^{\text {in }}}\right|^{2},
$$

for the spin- $\frac{1}{2}$ case (similar expressions for the case $s=$ $-\frac{1}{2}$ can be obtained by substituting equations (23)- (25) into the expressions above). The quantities $r_{s}$ and $t_{s}$ can be interpreted as reflection and transmission coefficients, as confirmed by calculations of energy fluxes [19]. (See the Appendix A for the pertinent calculations.) We can see that $t_{0}$ can assume negative values when $\delta<0$, i.e., when

$$
\omega-\frac{q Q}{r_{+}}<0
$$

Therefore, similarly to what happens for an uncharged scalar particle scattered by a Kerr black hole [23], a charged particle scattered by a RN black hole can effectively extract energy from it 24. This phenomenon, generically called superradiance [25], can be interpreted as a stimulated emission process [26] for certain particles. Note that the transmission coefficient for spin- $\frac{1}{2}$ fermions is always non-negative and, therefore, (electrical) superradiance is also impossible for such fermions [19], exactly in the same manner it occurs for the Kerr case [27].

\section{CHALLENGING THE WCCC}

We are now ready to investigate the validity of the WCCC when low energy spin- 0 and spin- $\frac{1}{2}$ particles tunnel into a near-extreme RN black hole. Before the scattering process starts, the black hole's mass $M$ and charge $Q$ satisfy the relation

$$
0<M^{2}-Q^{2} \ll M^{2} .
$$

As a result of the tunneling process, the black hole absorbs the particle total energy $\omega$, charge $q$ and the total angular momentum $L$. If we want to destroy the event horizon and create a naked singularity, the following relation must be satisfied

$$
f=(M+\omega)^{2}-(Q+q)^{2}-\frac{L^{2}}{(M+\omega)^{2}}<0 .
$$

In order to make this process possible even for zero angular momentum particles, we choose $Q$ and $q$ to have the same sign, which we assume, without loss of generality for the purposes here, to be positive. (The calculations for the general case are presented in the Appendix.) In the spinless case, the particle's energy cannot be arbitrarily small, otherwise superradiance occurs and, instead of tunneling into the black hole, the particle extracts energy and charge from it [24]. From Eq. (29), we conclude that $\omega$ must satisfy

$$
\omega>\frac{q Q}{r_{+}}
$$

if the particle is to be absorbed by the black hole. Choosing the incident particle to have zero angular momentum (i.e. $\ell=j=0$ and, therefore, $L=0$ ) in order to minimize any backreaction effect related to rotations, condition (33) above reduces simply to

$$
\omega<q-(M-Q)
$$

Note that, similarly to what happens in the classical process [4], if the black hole is initially extreme, i.e. $M=Q$, we have $r_{+}=M$ and it is impossible to find $\omega$ satisfying Eqs. (34) and (35) simultaneously. However, for a nearextreme black hole, if the particle's charge is chosen to be

$$
q>\frac{M-Q}{r_{+}-Q} r_{+}=\frac{r_{+}-Q}{2}
$$

then it is always possible to find energies $\omega$ for which the inequalities (34) and (35) are satisfied simultaneously. Notice that the restriction (36) is exactly the same obtained previously for the classical gedanken experiments involving charged test bodies, see [4, 7]. For the limit of near extreme black holes, one has

$$
q>q_{0}=\sqrt{\frac{M \varepsilon}{2}}+O(\varepsilon),
$$

where $M-Q=\varepsilon>0$. Let us consider now $\omega=$ $\left(q Q / r_{+}\right)+\delta_{0}$, with positive $\delta_{0}$. Expanding the expression for $f$ in the lowest orders for $\delta_{0}$ and $\varepsilon$, we obtain

$$
f=2(M+q) \delta_{0}-2 q \sqrt{\frac{2 \varepsilon}{M}}(M+q)+O\left(\delta_{0}^{2}, \varepsilon\right) .
$$

We conclude that if $\delta_{0}$ and $\varepsilon$ are sufficiently small and satisfy

$$
0<\delta_{0}<q \sqrt{\frac{2 \varepsilon}{M}},
$$

with $q>q_{0}$, then the condition (33) for the creation of a naked singularity is fulfilled. It is important we keep in these absorption processes the validity of the test field approximation, $\omega \ll M$ and $q \ll Q$, otherwise a myriad of backreaction effects could appear preventing effectively the absorption. Even though it is possible to keep the deviations of the test field approximation for scalar 
fields to a minimum, we will see that the situation for the fermions is still more favorable.

For the spin- $\frac{1}{2}$ case, superradiance is absent, and the particle's energy $\omega$ can, in principle, have any value greater than zero that the tunneling probability will remain positive even for arbitrarily small $q$, keeping in this way backreaction effects really to the minimum. Let us consider again $\ell=0$ (and, therefore, $L^{2}=s(s+1)=$ $3 / 4$ ). The first order expansion of $f$ in terms of $\omega$ and $\varepsilon$ is given by

$$
f \approx 2\left(M+\frac{3}{4 M^{3}}\right) \omega+2(M+q) \varepsilon-2 M q-q^{2}-\frac{3}{4 M^{2}} .
$$

The condition necessary to impose Eq. (33) and, consequently, create a naked singularity is

$$
0<\omega<\frac{2 M q+q^{2}+\frac{3}{4 M^{2}}-2(M+q) \varepsilon}{2 M+\frac{3}{2 M^{3}}} .
$$

In contrast with the scalar case, there is no minimal value for $q$ in order to assure the tunneling. Let us suppose that the spin- $\frac{1}{2}$ field be a small perturbation (both $\omega, q \ll M$ ) and that the black hole is large $(M \gg 1$, assuring in this way that total angular momentum $L^{2}=3 / 4$ of the fermion is also a small perturbation). In this approximation, Eq. (41) reads

$$
0<\omega<q-\varepsilon,
$$

whose interpretation is rather simple: the only requirement on the charge $q$ is that it must be enough to overcharge the black hole. For any charge capable of this, there are energies for which the incident particles might convert the black hole into a naked singularity. Spin- $\frac{1}{2}$ fields can elude the backreaction effects arising from the breakdown of the test field approximation in the most efficient way.

\section{A. Vacuum polarization and quantum statistics}

Notwithstanding, there is a point of concern for fermions, raised by Hod in Ref. 12]: the spontaneous polarization of the vacuum around a black hole and the Pauli exclusion principle. In order to properly introduce such effect, let's briefly review some aspects of particle production by black holes. In time-dependent spacetimes, e.g. the gravitational collapse of a spherical body, particle production occurs because the Hamiltonian describing the evolution of the field is time-dependent and, therefore a mixing of positive and negative frequencies can happen. Since particles and antiparticles are usually described by positive and negative frequencies, one then says that particle production has occured. However, the correct definition of particle/antiparticle states is given in terms of an appropriate pseudonorm (in order to be compatible with the (anti-)commutation relations). For example, for scalar fields the pseudonorm is naturally defined from the Klein-Gordon scalar product. When a mode has a positive norm, it is called a particle state; on the other hand, when a mode has negative norm, it is called an antiparticle state. Usually, this definition is equivalent to defining particle states with respect to the corresponding mode frequency. However, for charged black holes (and also for rotating ones) this is not the case. The eletromagnetic interaction between the field and the black hole makes it possible for a mode with given frequency $\omega$ to have positive norm in the vicinity of the black hole and negative norm far away from it, characterizing again particle production. In other words, the electric field is so strong in the vicinity of the black hole that a pair particle/antiparticle can be created [15, 28]. One of them escapes to infinity, since it has positive energy (as measured by an observer at infinity) and its charge has the same sign as the black hole's charge. The other particle (antiparticle), has negative energy and is absorbed by the black hole, discharging it and reducing its electromagnetic energy. The expected number of particles spontaneously emitted in each mode is [15, 29]

$$
\left\langle n_{\frac{1}{2}}\right\rangle=t_{\frac{1}{2}}\left[1+e^{x}\right]^{-1}, \quad x=\frac{\omega-q Q / r_{+}}{T_{\mathrm{bh}}},
$$

where $T_{\mathrm{bh}}$ is the temperature of the black hole and the transmission coefficient $t_{\frac{1}{2}}$ is given by expression A17. For extreme black holes $\left(T_{\mathrm{bh}}=0\right)$ only superradiant modes $\left(\omega<q Q / r_{+}\right)$are emitted. Back to the question of cosmic censorship, the interesting situation to be considered is that of a black hole immersed in a thermal radiation bath which obeys Fermi-Dirac statistics. Including spontaneous emission and pure scattering effects, the probability that one fermion is incident on the black hole without reflection is [29, 30]

$$
p_{\frac{1}{2}}=t_{\frac{1}{2}}\left[1+e^{-x}\right]^{-1} .
$$

Clearly, if the black hole is initially extreme, the probability that a low energy particle $\left(\omega<q Q / r_{+}\right)$enters the black hole vanishes. However, for a near-extreme black hole, the probability is, albeit small, always positive and, therefore, the possibility of creating a naked singularity really exists. Consider, for example, the case of a very low energy $(\omega M \ll 1)$ and low angular momentum $(\ell \ll q M)$ particle, incident on a near extreme black hole. Eq. (44) reduces, in this limit, to

$$
p_{\frac{1}{2}} \rightarrow e^{x},
$$

where expression A19) for the corresponding transmission coefficient $t_{\frac{1}{2}}$ was used. Note the similarity of this result with the classical gedanken experiments described in the introduction, in which a violation of the WCCC can only happen for near-extreme black holes (and not for extreme black holes).

It is also interesting to analyze the effect of vacuum polarization on the tunneling probability of scalar particles by considering a charged black hole immersed in a 
thermal radiation bath obeying Bose-Einstein statistics. In this case, including spontaneous emission, stimulated emission and pure scattering effects, the probability that $n$ scalar particles are incident on the black hole without reflection is 29, 30]

$$
p_{0}=t_{0}^{n} e^{x n} \frac{e^{x}-1}{\left(e^{x}-1+t_{0}\right)^{n+1}},
$$

where $t_{0}$ is given by expression (A17). Since $p_{0}$ is always positive for non-extreme black holes, even superradiant modes have a non-zero probability of being absorbed without any kind of emission/reflection by the black hole. Therefore, conditions (34) and (35) are replaced by

$$
0<\omega<n q-(M-Q)
$$

and we conclude that it is possible, in principle, to violate the WCCC using scalar particles with small charges $q$, even though the typical probabilities can be considerably small. In particular, for a very low energy $(\omega M \ll 1)$ and low angular momentum $(\ell \ll q M)$ particle, Eq. (46) reduces to

$$
p_{0} \rightarrow 2^{-(n+1)} e^{x n},
$$

where expression A19) for the corresponding transmission coefficient $t_{0}$ was used. Observe that one could, effectively, use several particles $(n>1)$ with $q<\varepsilon$ in order to create a naked singularity. However, the greatest probability (same order of magnitude of the spin- $\frac{1}{2}$ case) is obtained for a single particle $(n=1)$.

\section{CONCLUSION}

We investigated the possibility of violating the WCCC by quantum tunneling of charged spin- 0 and spin- $\frac{1}{2}$ particles into a RN black hole. If the black hole configuration is initially extreme $M=Q$, the tunneling of particles with enough charge to create a naked singularity is impossible, irrespective of the particle spin. This was also the case in classical gedanken experiments attempting to destroy the event horizon of a black hole [2, 4, 6]. However, for a near-extreme RN black hole, an incident particle can, in principle, be quantum mecanically absorbed and overcharge the black hole, destroying its event horizon. Besides, we obtained analytical results, in the small energy limit $(M \omega \ll 1)$, for the transmission coefficient of spin- 0 and spin- $\frac{1}{2}$ fields scattered by a charged black hole. As for the Kerr spacetime case, electrical superradiance makes more difficult the formation of a naked singularity by the absorption of scalar particles. The situation for the scalar field is similar to the classical gedanken experiments involving small charged bodies [4, 7]. However, for spin- $\frac{1}{2}$, superradiance is absent, and it is possible to choose the parameters of the particle in order to destroy the black hole's event horizon keeping to a minimum any backreaction effect related to the breakdown of the test field approximation. We also showed that vacuum polarization effects cannot be invoked to elude the creation of a naked singularity.

The quantum nature of the processes we are proposing here is fundamental for the violation of the WCCC. Indeed, Ref. [14] shows, in a very similar context, how different the quantum tunneling and its classical limit can be. Even though the particle propagates as a wave due to the wave-particle duality, it tunnels into the black hole as a single quantum. For instance, a possible way to include backreaction effects for scalar particles would be to solve the coupled Einstein-Klein-Gordon equations,

$$
\begin{array}{r}
\left(\nabla_{\mu}-i q A_{\mu}\right)\left(\nabla^{\mu}-i q A^{\mu}\right) \phi=0, \\
G_{\mu \nu}=8 \pi\left\langle T_{\mu \nu}\right\rangle,
\end{array}
$$

where $\left\langle T_{\mu \nu}\right\rangle$ is the vacuum expectation value for the stress energy tensor naturally assigned to the quantum scalar field $\phi$. However, the change from a black hole configuration to a naked singularity is certainly not a smooth process. Even this backreaction calculation would be insufficient to determine the fate of the black hole. A definite answer about the validity of the WCCC will only be possible when a complete quantum gravity theory becomes available.

\section{ACKNOWLEDGMENTS}

This work was supported by FAPESP and CNPq. The authors are grateful to G. Matsas, R.P. Macedo, R. Santarelli, T.P. Sotiriou, and D. Vanzella for enlightening discussions.

\section{Appendix A: Reflection and transmission coefficients}

In order to determine the reflection and transmission coefficients (29) and (30) in the small energy limit $M \omega \ll$ 1 of the radial master equation (17), we follow the same approach used in 31] for the Kerr case. The charges $q$ and $Q$ here are generic. By introducing

$$
x=\frac{r-r_{+}}{r_{+}-r_{-}},
$$

Eq. (17) can be written in the limit $M \omega \ll 1$ as 
$x^{2}(x+1)^{2} \frac{d^{2} R_{s}}{d x^{2}}+(s+1)(2 x+1) x(x+1) \frac{d R_{s}}{d x}+\left[k^{2} x^{4}+2(i s-q Q) k x^{3}+\left(q^{2} Q^{2}-\lambda_{s}\right) x(x+1)+G_{s} x+H_{s}\right] R_{s}=0$,

where

$$
\begin{aligned}
k & =\omega\left(r_{+}-r_{-}\right), \\
G_{s} & =\left(\frac{s}{2}-i \delta-i q Q\right)^{2}-\left(\frac{s}{2}+i \delta\right)^{2}, \\
H_{s} & =\left(\frac{s}{2}\right)^{2}-\left(\frac{s}{2}+i \delta\right)^{2} .
\end{aligned}
$$

In the limit $k x \ll 1$, the first two terms inside the square brackets in Eq. A2 can be ignored. The solution corresponding to an ingoing wave near the horizon is given by

$$
R_{s}=A_{s}(1+x)^{-s+i \delta+i q Q} x_{2}^{-s-i \delta} F_{1}(a, b ; c ;-x),
$$

where ${ }_{2} F_{1}(a, b ; c ; x)$ stands for the ordinary hypergeometric function,

$$
\begin{gathered}
a=\frac{1}{2}-s+i q Q-i \beta, \quad b=\frac{1}{2}-s+i q Q+i \beta, \\
c=1-s-2 i \delta, \quad \beta=\sqrt{q^{2} Q^{2}-\left(j+\frac{1}{2}\right)^{2}},
\end{gathered}
$$

and $A_{s}$ must be chosen so that Eqs. (23)-(25) are satisfied. In fact, comparing the asymptotic behavior near the horizon of Eq. (A6) with Eq. (22), we have

$$
Z_{s}^{\mathrm{tr}}=A_{s} r_{+}\left(r_{+}-r_{-}\right)^{\frac{3}{2} s+i \delta}
$$

If Eq. (25) is to be satisfied, we conclude that $A_{+\frac{1}{2}}$ and $A_{-\frac{1}{2}}$ must be related according to

$$
\frac{A_{+\frac{1}{2}}}{A_{-\frac{1}{2}}}=\frac{\sqrt{2}}{\lambda\left(r_{+}-r_{-}\right)}\left(\frac{1}{2}-2 i \delta\right)
$$

Once one of the parameters is arbitrarily chosen, the other one is automatically determined by the expression above. For the spin- 0 case, there is only one parameter, $A_{0}$, which can be chosen arbitrarily.

Considering now the limit $x \gg 1$, the last two terms inside the square brackets in Eq. (A2) can be dropped and $x+1$ can be simply replaced by $x$. The corresponding solution is

$$
R_{s}=C_{1} x^{-\frac{1}{2}-s+i \beta} e^{-i k x}{ }_{1} F_{1}\left(d_{1}, 1+2 i \beta ; 2 i k x\right)+C_{2} x^{-\frac{1}{2}-s-i \beta} e^{-i k x}{ }_{1} F_{1}\left(d_{2}, 1-2 i \beta ; 2 i k x\right),
$$

where ${ }_{1} F_{1}(a, b ; x)$ stands for the confluent hypergeometric function and $d_{1}$ and $d_{2}$ are given by

$$
d_{1}=\frac{1}{2}-s+i \beta-i q Q, \quad d_{2}=\frac{1}{2}-s-i \beta-i q Q .
$$

By matching the two solutions, i.e. Eqs. (A6) and (A11), in the overlap region $1 \ll x \ll 1 / k$, it is possible to determine the coefficients $C_{1}$ and $C_{2}$,

$$
C_{1}=A_{s} \frac{\Gamma(2 i \beta) \Gamma(c)}{\Gamma(b) \Gamma(c-a)}, \quad C_{2}=A_{s} \frac{\Gamma(-2 i \beta) \Gamma(c)}{\Gamma(a) \Gamma(c-b)}
$$

Now, comparing the asymptotic form of the confluent hypergeometric functions ${ }_{1} F_{1}(a, b ; x)$ with Eq. (21), we find

$$
\frac{Z_{s}^{\text {in }}}{\left(r_{+}-r_{-}\right)^{1-i q Q}}=C_{1} \frac{\Gamma(1+2 i \beta)(-2 i k)^{-\frac{1}{2}+s-i \beta+i q Q}}{\Gamma\left(\frac{1}{2}+s+i \beta+i q Q\right)}+C_{2} \frac{\Gamma(1-2 i \beta)(-2 i k)^{-\frac{1}{2}+s+i \beta+i q Q}}{\Gamma\left(\frac{1}{2}+s-i \beta+i q Q\right)} .
$$

Using Eqs. (A9) and (A14), we calculate

$$
\left|\frac{Z_{s}^{\mathrm{tr}}}{Z_{s}^{\text {in }}}\right|^{2}=\frac{r_{+}^{2}\left(r_{+}-r_{-}\right)^{3 s-2} e^{-\pi q Q}(2 k)^{1-2 s}}{|\Gamma(c)|^{2}|F(\beta)+F(-\beta)|^{2}},
$$

where

$$
F(\beta)=\frac{\Gamma(2 i \beta) \Gamma(1+2 i \beta) e^{-\frac{\pi \beta}{2}-i \beta \log (2 k)}}{\Gamma(b) \Gamma(c-a) \Gamma\left(\frac{1}{2}+s+i q Q+i \beta\right)} .
$$


Plugging Eq. (A15) into the expressions (29) and (30), we obtain the formula for the transmission coefficient,

$$
t_{s}=\frac{e^{-\pi q Q}(2 \delta)^{1-2|s|}}{|\Gamma(1-|s|-2 i \delta)|^{2}|F(\beta)+F(-\beta)|^{2}} .
$$

When $j+1 / 2>|q Q|, \beta$ is purely imaginary, $\beta=i \gamma$, with $\gamma>0$. In the limit of small energies, $k \rightarrow 0$, we can write

$$
t_{s}=\frac{e^{-\pi q Q}(2 \delta)^{1-2|s|}\left|\Gamma\left(\frac{1}{2}+|s|+\gamma+i q Q\right) \Gamma\left(\frac{1}{2}-|s|+\gamma+i q Q\right) \Gamma\left(\frac{1}{2}+\gamma-i q Q-2 i \delta\right)\right|^{2}}{|\Gamma(1-|s|-2 i \delta)|^{2}[\Gamma(2 \gamma) \Gamma(1+2 \gamma)]^{2}}(2 k)^{2 \gamma}
$$

plus terms of order $O\left(k^{4 \gamma}\right)$ or higher. A similar result was obtained by Gibbons for massive scalar particles in the particular case $|q Q| \ll 1$, see Ref. [15]. Sampaio considered both cases of spin- 0 and spin- $\frac{1}{2}$ particles, also in the same limit, in Ref. [16]. However, when $j+1 / 2<|q Q|, \beta$ becomes a real number and, therefore, the transmission coefficient $t_{s}$ becomes a constant part plus an oscillating term in $k$. In the extreme case $j+1 / 2 \ll|q Q|$, the constant part dominates. When the charge of the black hole and the charge of the particle have the same sign (electric repulsion), the transmission coefficient reduces to

$$
t_{s}=(4|s|-1)+O\left(e^{-\pi q Q}\right)
$$

On the other hand, when the charges have opposite signs, the transmission coefficient is given by

$$
t_{s}=1-\frac{\pi}{|q Q|}\left(j+\frac{1}{2}\right)^{2}+O\left(\frac{1}{q^{2} Q^{2}}\right)
$$

The fact that the transmission coefficient approaches unity in this case is not suprising since the interaction between the particle and the black hole is attractive. However, interesting results arise when $q Q>0$. First, for spin- $\frac{1}{2}$ particles, even though the electromagnetic force is repulsive, the transmission coefficient approaches unity. Second, for scalar particles the transmission coefficient approaches -1 (and the reflection coefficient approaches 2 ), characterizing a situation of extreme superradiance. These extreme effects are caused by the fact that the electromagnetic term is the dominant term in the coefficient $q^{2} Q^{2}-\lambda_{s}$ of $x(x+1)$ in Eq. A2 and, no matter what the relative sign between the charges is, it always contributes with a positive sign.
[1] R. Penrose, Riv. Nuovo Cim. 1, 252 (1969).

[2] R. M. Wald, Annals of Physics 82, 548 (1974).

[3] D. G. Boulware, Phys. Rev. D 8, 2363 (1973); T. Needham, ibid. 22, 791 (1980); R. M. Wald, "Gravitational collapse and cosmic censorship," (1997), arXiv:gr-qc/9710068

[4] V. E. Hubeny, Phys. Rev. D 59, 064013 (1999), arXiv:gr-qc/9808043.

[5] S. Hod, Phys. Rev. D 66, $024016 \quad$ (2002), arXiv:gr-qc/0205005

[6] T. Jacobson and T. P. Sotiriou, Phys. Rev. Lett. 103, 141101 (2009), arXiv:0907.4146

[7] A. Saa and R. Santarelli, Phys. Rev. D 84, 027501 (2011), arXiv:1105.3950.

[8] E. Berti, V. Cardoso, L. Gualtieri, F. Pretorius, and U. Sperhake, Phys. Rev. Lett. 103, 239001 (2009), arXiv:0911.2243 M. Bouhmadi-Lopez, V. Cardoso, A. Nerozzi, and J. V. Rocha, Phys. Rev. D 81, 084051 (2010), arXiv:1003.4295, T. Jacobson and T. P. Sotiriou, "Might black holes reveal their inner secrets?." (2010), arXiv:1006.1763 G. Chirco, S. Liberati, and T. P. Sotiriou, ibid. 82, 104015 (2010), arXiv:1006.3655; J. V. Rocha and V. Cardoso, ibid. 83, 104037 (2011), arXiv:1102.4352; S. Isoyama, N. Sago, and T. Tanaka, "Cosmic censorship in overcharging a Reissner-Nordström black hole via charged particle absorption," (2011), arXiv:1108.6207.

[9] S. Hod, Phys. Rev. Lett. 100, 121101 (2008), arXiv:0805.3873

[10] E. Barausse, V. Cardoso, and G. Khanna, Phys. Rev. Lett. 105, 261102 (2010), arXiv:1008.5159. "Testing the Cosmic Censorship Conjecture with point particles: the effect of radiation reaction and the self-force," (2011), arXiv:1106.1692

[11] G. E. A. Matsas and A. R. R. da Silva, Phys. Rev. Lett. 99, 181301 (2007), arXiv:0706.3198.

[12] S. Hod, Phys. Lett. B 668, 346 (2008), arXiv:0810.0079.

[13] M. Richartz and A. Saa, Phys. Rev. D 78, 081503 (2008), arXiv:0804.3921

[14] G. E. A. Matsas, M. Richartz, A. Saa, A. R. R. da Silva, and D. A. T. Vanzella, Phys. Rev. D 79, 101502 (2009), arXiv:0905.1077

[15] G. W. Gibbons, Comm. Math. Phys. 44, 245 (1975).

[16] M. O. Sampaio, JHEP 0910, 008 (2009), arXiv:0907.5107

[17] T. Nakamura and H. Sato, Phys. Lett. B 61, 371 (1976).

[18] J. Stewart, Advanced General Relativity (Cambridge University Press, 1991). 
[19] C. H. Lee, Phys. Lett. B 68, 152 (1977).

[20] D. N. Page, Phys. Rev. D 14, 1509 (1976); 16, 2402 (1977).

[21] S. A. Teukolsky, Phys. Rev. Lett. 29, 1114 (1972).

[22] J. N. Goldberg, A. J. Macfarlane, E. T. Newman, F. Rohrlich, and E. C. G. Sudarshan, J. Math. Phys. 8, 2155 (1967).

[23] C. W. Misner, Phys. Rev. Lett. 28, 994 (1972); W. Unruh, Phys. Rev. D 10, 3194 (1974).

[24] J. D. Bekenstein, Phys. Rev. D 7, 949 (1973).

[25] J. D. Bekenstein and M. Schiffer, Phys. Rev. D 58, 064014 (1998), arXiv:gr-qc/9803033. M. Richartz, S. We- infurtner, A. J. Penner, and W. G. Unruh, 80, 124016 (2009), arXiv:0909.2317

[26] R. M. Wald, Phys. Rev. D 13, 3176 (1976).

[27] W. G. Unruh, Phys. Rev. Lett. 31, 1265 (1973).

[28] S. P. Kim and D. N. Page, Nuovo Cim. B 120, 1193 (2005), arXiv:gr-qc/0401057

[29] J. D. Bekenstein and A. Meisels, Phys. Rev. D 15, 2775 (1977).

[30] P. Panangaden and R. M. Wald, Phys. Rev. D 16, 929 (1977).

[31] A. A. Starobinskii and S. M. Churilov, Sov. Phys. JETP 38, 1 (1974); D. N. Page, Phys. Rev. D 13, 198 (1976). 